\title{
ERROR ANALYSIS FOR THE LOCAL ALGORITHM OF THE TWO-LEVEL METHOD IN SPARSE TIME-FREQUENCY ANALYSIS
}

\author{
Wen Wang \\ Department of Mathematics, Jinan University, Guangzhou, 510632, P. R. China
}

\begin{abstract}
In this paper, we give some theoretical discussion on the local algorithm of the two level method, which is shown to be efficient in data experiments. Under the assumption of scale separation, we show that the errors between the local optimal solution and the real intrinsic mode function (IMF) in the subintervals are still $O\left(\sqrt{\left(N_{p} d\right)^{-1}+\varepsilon}\right)$.

Keywords: two-level method, multi-scale data, nonlinear matching pursuit, sparse time-frequency representation.
\end{abstract}

\footnotetext{
${ }^{*}$ Corresponding author.

E-mail address: wangwenloki@163.com (Wen Wang).
}

Copyright (C) 2020 Scientific Advances Publishers 2020 Mathematics Subject Classification: 90C26.

Submitted by Jianqiang Gao.

Received February 26, 2020 


\section{Introduction}

In recent years, many researchers have focused on how to accurately extract hidden information from big data by using algorithms and models, so as to discover hidden problems or predict future development trends.

In our lives, stationary signals are mostly manufactured, most signals in nature are non-stationary [15]. Non-stationary signal whose distribution parameters or distribution law will vary because of the time's change, that is, the statistical characteristics change with time, such as biomedical signals, voice signals, and radar signals etc. Frequency is a very important feature of non-stationary signal, because they contain many important information of practical significance. Fourier transform can express the signal into the spectrum domain, so as to obtain the accurate information of the signal. It still has inherent defect for non-stationary signal, it only shows the frequency components for non-stationary signal, but it can't show the time of each frequency components' emergence. Therefore signals with the same frequency components but at different times may have the same spectrogram, this's not conducive to the research of many non-stationary signals.

In order to make up for the limitation of Fourier transform in our practical application, many scholars proposed Joint time-frequency analysis (JTFA) to analyze nonlinear and non-stationary signals. JTFA provides the joint distribution information of time domain and frequency domain, and clearly describes the relationship between signal frequency and time, which is beneficial to further processing of signal and extracting effective information. The common time-frequency analysis methods include Wingner-Ville distribution [1], Wavelet transform (WT) [2], Hilbert-Huang transform (HHT) [3], and Empirical mode decomposition (EMD) [4], etc. These methods use linear transformations such as translation, scaling, and distortion to change the shape and position of nonlinear non-stationary signals on the time-frequency distribution plane, so that we can extract the effective information of the frequency and amplitude more effectively, these are beneficial to the processing of the non-stationary signal. Van der Pol [5] and Gabor [6] 
propose another important approach of study instantaneous frequency, named Analytic signal (AS) method, they use Hilbert transform to constitute the real part and imaginary part associated with no negative frequency component of the complex function, the method is suitable for the monocomponent signals.

For multi-component signals, Empirical mode decomposition (EMD) proposed by Huang et al. [4] has many applications (see [7, 8]). It can decompose signal into a finite number of intrinsic mode functions (IMFs) [4] with a small residual.

$$
f(t)=\sum_{k=1}^{M} a_{k}(t) \cos \theta_{k}(t)+r(t), \quad t \in \mathbb{R} .
$$

An important feature of these IMFs is that they contain information of physical information, such as trends and instantaneous frequencies, and give a physical representation of the Hilbert spectrum. The disadvantage of the EMD is sensitivity to noise. Obviously, the local extremum of the signal is very sensitive to noise disturbance. Although EMD has made great achievements in many fields. However, its accuracy and applicability lack the support of mathematical theories. In detail, the expression form of IMF and the screening process' convergence are still worth studying. Hou and Shi found EMD can be seen as a sparse representation of the IMFs' dictionary methods. And they solve the following nonlinear optimization problem (P1) for the sparsest decomposition:

$$
\begin{aligned}
& \underset{\left(a_{k}\right)_{1 \leq k \leq M,}}{\operatorname{Minimize}\left(\theta_{k}\right)_{1 \leq k \leq M}^{M},} \\
& \text { subject to }\left\|f-\sum_{k=1}^{M} a_{k} \cos \theta_{k}\right\|_{L^{2}} \leq \varepsilon, \quad a_{k} \cos \theta_{k} \in \mathcal{D},
\end{aligned}
$$

where $\mathcal{D}$ is the dictionary which includes all IMFs. Hou and Shi are inspired by compressed sensing, this paper puts forward Data driven time-frequency analysis (DDTFA) method (see [9, 10]), what research the 
sparse representation of multi-scale signal on multi-scale basis. The multi-scale basis is adapted to the signal rather than determined a priori. They also demonstrate detailedtly the convergence of the method in [11].

Definition 1.1 (Scale-separation). One function $f(t)=a(t) \cos \theta(t)$ is said to satisfy a scale separation property with a separation factor $\varepsilon>0$ if $a(t)$ and $\theta(t)$ satisfy the following conditions:

$$
\begin{aligned}
& a(t) \in C^{1}(\mathbb{R}), \theta(t) \in C^{2}(\mathbb{R}), \inf _{t \in \mathbb{R}} \theta^{\prime}(t)>0, \\
& \frac{\sup _{t \in \mathbb{R}} \theta^{\prime}(t)}{\inf _{t \in \mathbb{R}} \theta^{\prime}(t)} \leq M^{\prime}<+\infty,\left|\frac{a^{\prime}(t)}{\theta^{\prime}(t)}\right| \leq \varepsilon,\left|\frac{\theta^{\prime \prime}(t)}{\left(\theta^{\prime}(t)\right)^{2}}\right| \leq \varepsilon, \quad \forall t \in \mathbb{R} .
\end{aligned}
$$

In the above definition, the condition that $\theta^{\prime}(t)>0$ makes sure the instantaneous frequency have a physical meaning, the last two conditions means that both the envelope $a(t)$ and the frequency $\theta^{\prime}(t)$ are more smooth than $\cos \theta(t)$.

With the above definition, we put all the IMFs satisfying scale separation together:

$$
\mathcal{D}_{\varepsilon}:=\left\{a(t) \cos \theta(t):(a, \theta) \in U_{\varepsilon}\right\},
$$

where

$$
U_{\varepsilon}=\left\{(a, \theta): a>0, \theta^{\prime}>0 ; \frac{\sup _{t \in \mathbb{R}} \theta^{\prime}(t)}{\inf _{t \in \mathbb{R}} \theta^{\prime}(t)} \leq M^{\prime}<+\infty,\left|\frac{a^{\prime}(t)}{\theta^{\prime}(t)}\right| \leq \varepsilon,\left|\frac{\theta^{\prime \prime}(t)}{\left(\theta^{\prime}(t)\right)^{2}}\right| \leq \varepsilon\right\} .
$$

Definition 1.2 (Well-separated signal). A signal $f: \mathbb{R} \rightarrow \mathbb{R}$ is said to be well separated with separation factor $\varepsilon$ and frequency ratio $d$ can be expressed as

$$
f(t)=\sum_{k=1}^{M} a_{k}(t) \cos \theta_{k}(t)+r(t)
$$


where all $f_{k}(t)=a_{k}(t) \cos \theta_{k}(t)$ satisfy the scale separation property with separation factor $\varepsilon, r(t)=O(\varepsilon)$, and their phase function $\theta_{k}(t)$ satisfy

$$
\theta_{k}^{\prime}(t) \geq d \theta_{k-1}^{\prime}(t), \quad \forall t \in \mathbb{R}
$$

and $d>1, d-1=O(1)$.

Liu et al. analyzed the uniqueness of the solution of the following nonlinear optimization problem (P2) in nonlinear matching pursuit (NMP) [14] of EMD (see [12]):

$$
\begin{aligned}
& \text { Minimize } p(a, \theta):=\|f(t)-a(t) \cos \theta(t)\|_{L^{2}}^{2} \\
& \text { subject to }(a, \theta) \in U_{\varepsilon} .
\end{aligned}
$$

They also proved that the solution of the optimal problem is unique up to the error determined by the scale-separation property under the premise of the well-separated signals. Meanwhile it demonstrated the error between the local optimal solution and global optimal solution received from the optimal problem. Yang [16] noticed that the time window may not contain integer signal period in practical problems, and proved that, if $\theta^{\prime}(t)$ is close to $\theta_{l}^{\prime}(t)$, then relative error from the local optimal solution $(a, \theta)$ to $\left(a_{l}, \theta_{l}\right)$ is $O\left(\sqrt{\left(\eta_{l} d\right)^{-1}+\varepsilon}\right)$, where $\eta_{l}$ is the signal's period numbers.

The instantaneous frequency of signal may vary a lot, the constant initialization as initial solution probably cause mode mixing. The finite time range may introduce end effect. These all affect the accuracy of results. To alleviate these problems, Liu et al. raised two level method [13] to get the sparse time-frequency. This method use a local algorithm to get an initial solution, then use this solution as an initial in the global algorithm to receive an accurate global IMF and instantaneous frequency. In addition, they propose an algorithm to reduce the end effects. 


\section{The Local Algorithm in Two-Level Method}

Under the assumption that the instantaneous frequency is a smooth function locally, we can approximate the instantaneous frequency as a constant locally. Based on data-driven time frequency analysis method, Liu et al. proposed a two-level algorithm to obtain sparse time-frequency decomposition of multi-scale data, which mainly includes three parts: local algorithm, global algorithm, and algorithm (End Effect). In this section, we review the local algorithm briefly.

In the local algorithm, we first solve the problem (P2) in the entire time interval to get one solution. This solution may not be a correct IMF. But it could help us to determine a small interval, denoted by $\left[t_{0, l}, t_{0, r}\right]$, on which we are sure that the solution $g_{0}^{\text {local }}=a_{0}^{\text {local }} \cos \theta_{0}^{\text {local }}$ of (P2) in this interval is the part of some $\operatorname{IMF} a_{l}(t) \cos \theta_{l}(t)$, with about $N_{p}$ periods. Next, we select $\left[t_{1, l}, t_{0}^{\prime}\right] \subset\left[t_{0, l}, t_{0, r}\right]$ in the following:

$$
\begin{aligned}
& t_{1, l}=\arg \min _{\substack{t \in\left[t_{0, l}, t_{0, r}\right], \theta_{0} \text { local }(t) / \pi \in \mathbb{Z}}}\left|t-\left[t_{0, l}+\nu\left(t_{0, r}-t_{0, l}\right)\right]\right|, \\
& \theta_{0}^{\text {local }}(t) / \pi \in \mathbb{Z} \\
& t_{0}^{\prime}=t_{0, r}-\nu\left(t_{0, r}-t_{0, l}\right),
\end{aligned}
$$

where $\nu=1 / 3$, and let

$$
t_{1, r}=t_{1, l}+2 \pi N_{p} \cdot \frac{\left[t_{0, r}-\nu\left(t_{0, r}-t_{0, l}\right)\right]-\left[t_{0, l}+\nu\left(t_{0, r}-t_{0, l}\right)\right]}{\theta\left[t_{0, r}-\nu\left(t_{0, r}-t_{0, l}\right)\right]-\theta\left[t_{0, l}+\nu\left(t_{0, r}-t_{0, l}\right)\right]} .
$$

Then use the information of $g_{0}^{\text {local }}$, we solve the following weighted problem:

$$
\left(a_{1}^{\text {local }}, \theta_{1}^{\text {local }}\right)=\arg \min _{a, \theta}\|f-a \cos \theta\|_{L_{\left[t_{1, l}, t_{1, r}\right]}^{2}}^{2}+\omega_{1}\left\|g_{0}^{\text {local }}-a \cos \theta\right\|_{L_{\left[t_{1, l}, t_{0}^{\prime}\right]}^{2}}^{2}
$$

subject to $a \in V(\theta), \theta^{\prime} \geq 0$. 
Again we use the new solution on $\left[t_{1, l}, t_{1, r}\right]$ to determine the next subinterval and solve (P3) on it. Repeat this procedure until the whole time interval is covered by the subintervals, and we connect all the solutions to get a good initial of the problem (P2) to the real IMF $a_{l}(t) \cos \theta_{l}(t)$.

\section{Error Analysis for the Local Algorithm}

For the signal satisfying the scale property, the two-level algorithm makes improvement of the mode mixing and end effect of time-frequency analysis. In local algorithm, we apply the following problem (P4) to extend the subinterval to obtain a new initial solution:

$$
\begin{gathered}
\text { Minimize } s(a, \theta)=\|f-a \cos \theta\|_{L_{\left[t_{1, l}, t_{1, r}\right]}^{2}}^{2}+\omega_{1}\left\|g_{0}^{\text {local }}-a \cos \theta\right\|_{L_{\left[t_{1}, l, t_{0}^{\prime}\right]}^{2}}^{2} \\
\text { subject to }(a, \theta) \in U_{\varepsilon} .
\end{gathered}
$$

And initial solution is applied to the global algorithm. The weight problem solution error aroused our attention, in this section, we will discuss the errors caused by problem (P4) of the local optimal solution and the global optimal solution, respectively.

Lemma 3.1. Let $f(t)$ be a function satisfying the scale separation properties separation factor $\varepsilon$ and frequency ratio $d$ as defined in Definition 1.2:

$$
f(t)=\sum_{k=1}^{M} a_{k}(t) \cos \theta_{k}(t)+r(t), a_{k} \cos \theta_{k} \in U_{\varepsilon}, \quad a_{k}(t)=O(1), r(t)=O(\varepsilon) .
$$

If

$$
s(a, \theta) \leq s\left(a_{l}, \theta_{l}\right)
$$


where $s(a, \theta)$ is given in (P3), then we have

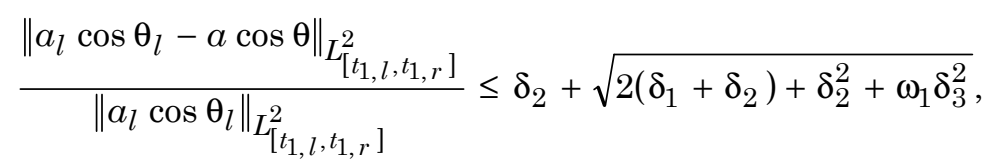

where

$$
\begin{aligned}
& \delta_{1}=\sum_{k \neq l} \frac{\left|\left\langle a_{l} \cos \theta_{l}, a_{k} \cos \theta_{k}\right\rangle\right|}{\left\|a_{l} \cos \theta_{l}\right\|_{L^{2}}\left\|a_{k} \cos \theta_{k}\right\|_{L^{2}}} \frac{\left\|a_{k} \cos \theta_{k}\right\|_{L^{2}}}{\left\|a_{l} \cos \theta_{l}\right\|_{L^{2}}}+\frac{\|r(t)\|_{L^{2}}}{\left\|a_{l} \cos \theta_{l}\right\|_{L^{2}}}, \\
& \delta_{2}=\sum_{k \neq l} \frac{\left|\left\langle a \cos \theta_{l}, a \cos \theta_{k}\right\rangle\right|}{\|a \cos \theta\|_{L^{2}}\left\|a_{k} \cos \theta_{k}\right\|_{L^{2}}} \frac{\left\|a_{k} \cos \theta_{k}\right\|_{L^{2}}}{\left\|a_{l} \cos \theta_{l}\right\|_{L^{2}}}+\frac{\|r(t)\|_{L^{2}}}{\left\|a_{l} \cos \theta_{l}\right\|_{L^{2}}}, \\
& \delta_{3}=\frac{\left\|g_{0}^{l o c a l}-a \cos \theta\right\|_{L_{\left[t_{1}, t_{2}\right]}^{2}}^{2}}{\left\|a_{l} \cos \theta_{l}\right\|_{L_{\left[t_{1}, t_{3}\right]}^{2}}^{2}}
\end{aligned}
$$

Proof. First, by the inequality (9) and the definition of $s$ in (P4), we have $0 \geq s(a, \theta)-s\left(a_{l}, \theta_{l}\right)$

$$
\begin{aligned}
& =\|f-a \cos \theta\|_{L_{\left[t_{1}, t_{3}\right]}^{2}}^{2}+\omega_{1}\left\|g_{0}^{\text {local }}-a \cos \theta\right\|_{L_{\left[t_{1}, t_{2}\right]}^{2}}^{2}-\left\|f-a_{l} \cos \theta_{l}\right\|_{L_{\left[t_{1}, t_{3}\right]}^{2}}^{2} \\
& -\omega_{1}\left\|g_{0}^{\text {local }}-a_{l} \cos \theta_{l}\right\|_{L_{\left[t_{1}, t_{2}\right]}^{2}}^{2} \\
& \geq\left\|a_{l} \cos \theta_{l}-a \cos \theta\right\|_{L^{2}\left[t_{1}, t_{3}\right]}^{2}+2\left\langle f-a_{l} \cos \theta_{l}, a_{l} \cos \theta_{l}-a \cos \theta\right\rangle_{L_{\left[t_{1}, t_{3}\right]}^{2}} \\
& +\omega_{1}\left\|g_{0}^{\text {local }}-a \cos \theta\right\|_{L_{\left[t_{1}, t_{2}\right]}^{2}}^{2} \\
& -\omega_{1}\left\|g_{0}^{\text {local }}-a_{l} \cos \theta_{l}\right\|_{L_{\left[t_{1}, t_{2}\right]}^{2}}^{2},
\end{aligned}
$$


where $t_{1}=t_{1, l}, t_{2}=t_{0}^{\prime}, t_{3}=t_{1, r}$. It is easy to see that

$$
\mid\left\langle a_{l} \cos \theta_{l}, \sum_{k \neq l} a_{k} \cos \theta_{k}+r(t)\right\rangle \leq \delta_{1}\left\|a_{l} \cos \theta_{l}\right\|_{L^{2}}^{2},
$$

and

$$
\begin{aligned}
& \quad\left|\left\langle a \cos \theta, \sum_{k \neq l} a_{k} \cos \theta_{k}+r(t)\right\rangle\right| \leq \delta_{2}\|a \cos \theta\|_{L^{2}}\left\|a_{l} \cos \theta_{l}\right\|_{L^{2}} \\
& \quad \leq \delta_{2}\left\|a_{l} \cos \theta_{l}-a \cos \theta\right\|_{L^{2}} \cdot\left\|a_{l} \cos \theta_{l}\right\|_{L^{2}}+\delta_{2}\left\|a_{l} \cos \theta_{l}\right\|_{L^{2}}^{2} .
\end{aligned}
$$

Then we have

$$
\begin{gathered}
0 \geq\left\|a_{l} \cos \theta_{l}-a \cos \theta\right\|_{L_{\left[t_{1}, t_{3}\right]}^{2}}^{2}-2 \delta_{2}\left\|a_{l} \cos \theta_{l}-a \cos \theta\right\|_{L_{\left[t_{1}, t_{3}\right]}^{2}}^{2}\left\|a_{l} \cos \theta_{l}\right\|_{L^{2}\left[t_{1}, t_{3}\right]} \\
-2\left(\delta_{1}+\delta_{2}\right)\left\|a_{l} \cos \theta_{l}\right\|_{L_{\left[t_{1}, t_{3}\right]}^{2}}^{2}-\omega_{1}\left\|g_{0}^{\text {local }}-a_{l} \cos \theta_{l}\right\|_{L_{\left[t_{1}, t_{2}\right]}^{2}}^{2}
\end{gathered}
$$

Divide both sides of this inequality by $\left\|a_{l} \cos \theta_{l}\right\|_{L_{\left[t_{1}, t_{3}\right]}^{2}}^{2}$, we obtain

$0 \geq \kappa^{2}-2 \delta_{2} \kappa-2\left(\delta_{1}+\delta_{2}\right)-\omega_{1} \delta_{3}^{2}=\left(\kappa-\delta_{2}\right)^{2}-\left[2\left(\delta_{1}+\delta_{2}\right)+\delta_{2}^{2}+\omega_{1} \delta_{3}^{2}\right]$,

with

$$
\kappa=\frac{\left\|a_{l} \cos \theta_{l}-a \cos \theta\right\|_{L_{\left[t_{1}, t_{3}\right]}^{2}}}{\left\|a_{l} \cos \theta_{l}\right\|_{L_{\left[t_{1}, t_{3}\right]}^{2}}^{2}}
$$

This implies (10). We complete the proof. 
Theorem 3.2. Under the assumptions of Theorem 3.1, and suppose there exist $\alpha \in[1, d)$ such that

$$
\alpha^{-1} \theta_{l}^{\prime}(t) \leq \theta^{\prime}(t) \leq \alpha \theta_{l}^{\prime}(t), \quad \forall t \in\left[t_{1, l}, t_{1, r}\right]
$$

Then we have

$$
\frac{\left\|a_{l} \cos \theta_{l}-a \cos \theta\right\|_{L_{\left[t_{1, l}, t_{1, r}\right]}^{2}}}{\left\|a_{l} \cos \theta_{l}\right\|_{L_{\left[t_{1, l}, t_{1, r}\right]}^{2}}}=O\left(\sqrt{\left(N_{p} d\right)^{-1}+\varepsilon}\right) .
$$

Proof. It follows from condition $r(t)=O(\varepsilon),(2.23)$ and (2.24) in [16] that for $1 \leq k \neq l \leq M$, we have

$$
\delta_{1}=O\left(\left(N_{p} d\right)^{-1}+\varepsilon\right), \quad \delta_{2}=O\left(\left(N_{p} d\right)^{-1}+\varepsilon\right) .
$$

Together with $g_{0}^{\text {local }}$ is a local minimizer of (P2) and Theorem 2.1 in [16], we know that

$$
\delta_{3}=O\left(\sqrt{\left(N_{p} d\right)^{-1}+\varepsilon}\right) .
$$

Substituting (18) and (19) into (11), we obtain (17).

From the above estimation, we see the following facts:

(1) The error between $a(t) \cos \theta(t)$ and $a_{l}(t) \cos \theta_{l}(t)$ may be greater than the error in the case that $\omega_{1}=0$. The reason is that $\delta_{3}$, the error between $g_{0}^{\text {local }}$ and $a_{l}(t) \cos \theta_{l}(t)$ may make more error in the problem (P4).

(2) The error is still $O\left(\sqrt{\left(N_{p} d\right)^{-1}+\varepsilon}\right)$. So we can assure that the error would not increase rapidly from one subinterval to the next subinterval. Therefore we could obtain an approximation of $a_{l}(t) \cos \theta_{l}(t)$ on the whole time interval in the level of $O\left(\sqrt{\left(N_{p} d\right)^{-1}+\varepsilon}\right)$. 


\section{Conclusion}

We estimated the error for the optimization problem in the local algorithm of the two level method in sparse time-frequency decomposition. The error we obtained gave some theoretical assurence that the local algorithm is efficient in searching the correct IMF. Our next work is to estimate the error between the global optimal solution and the real IMF.

\section{References}

[1] P. Flandrin, Time-Frequency/Time-Scale Analysis, Academic Press, San Diego, 1999.

[2] S. Mallat, A Wavelet Tour of Signal Processing: The Sparse Way, 3rd Edition, Amsterdam: Elsevier/Academic Press, 2009.

[3] N. E. Huang, Z. Wu and S. R. Long, Hilbert-Huang Transform, 2008.

DOI: https://doi.org/10.4249/scholarpedia.2544

[4] N. E. Huang, Z. Shen, S. R. Long, M. C. Wu, H. H. Shih, Q. Zheng, N. C. Yen, C. C. Tung and H. H. Liu, The empirical mode decomposition and the Hilbert spectrum for nonlinear and non-stationary time series analysis, Proceedings of the Royal Society, Series A: Mathematical, Physical and Engineering Sciences 454(1971) (1998), 903-995.

DOI: https://doi.org/10.1098/rspa.1998.0193

[5] B. Van der Pol, The fundamental principles of frequency modulation, Journal of the Institution of Electrical Engineers, Part III: Radio and Communication Engineering 93(23) (1946), 153-158.

DOI: https://doi.org/10.1049/ji-3-2.1946.0024

[6] D. Gabor, Theory of communication, Journal of IEE 93(26) (1946), 429-457.

[7] E. J. Candes, J. Romberg and T. Tao, Robust uncertainty principles: Exact signal reconstruction from highly incomplete frequency information, IEEE Transactions on Information Theory 52(2) (2006), 489-509.

DOI: https://doi.org/10.1109/tit.2005.862083

[8] E. J. Candes and T. Tao, Near-optimal signal recovery from random projections: Universal encoding strategies, IEEE Transactions on Information Theory 52(12) (2006), 5406-5425.

DOI: https://doi.org/10.1109/TIT.2006.885507 
[9] T. Y. Hou and Z. Q. Shi, Adaptive data analysis via sparse time-frequency representation, Advances in Adaptive Data Analysis 3(1-2) (2011), 1-28.

DOI: https://doi.org/10.1142/S1793536911000647

[10] T. Y. Hou and Z. Q. Shi, Data-driven time-frequency analysis, Applied and Computational Harmonic Analysis 35(2) (2013), 284-308.

DOI: https://doi.org/10.1016/j.acha.2012.10.001

[11] T. Y. Hou, Z. Q. Shi and P. Tavallali, Convergence of a data-driven time-frequency analysis method, Applied and Computational Harmonic Analysis 37(2) (2014), 235-270.

DOI: https://doi.org/10.1016/j.acha.2013.12.004

[12] C. G. Liu, Z. Q. Shi and T. Y. Hou, On the uniqueness of sparse time-frequency representation of multiscale data, Multiscale Modeling \& Simulation 13(3) (2015), $790-811$.

$$
\text { DOI: https://doi.org/10.1137/141002098 }
$$

[13] C. G. Liu, Z. Q. Shi and T. Y. Hou, A two-level method for sparse time-frequency representation of multiscale data, Science China Mathematics 60(10) (2017), 1733-1752.

DOI: https://doi.org/10.1007/s11425-016-9088-9

[14] T. Y. Hou and Z. Q. Shi, Sparse time-frequency representation of nonlinear and nonstationary data, Science China Mathematics 56(12) (2013), 2489-2506.

DOI: https://doi.org/10.1007/s11425-013-4733-7

[15] R. L. Smith, Nonlinear and Nonstationary Signal Processing, Cambridge University Press, 2000.

[16] Q. Yang, Error analysis for sparse time-frequency decomposition of non-integer period sampling signals, Journal of Information and Computing Science 14(1) (2019), 25-34. 